\title{
Formation of single-moon systems around gas giants
}

\author{
Yuri I. Fujii ${ }^{1,2}$ and Masahiro Ogihara ${ }^{3}$ \\ 1 Institute for Advanced Research, Nagoya University, Furo-cho, Chikusa-ku, Nagoya, Aichi 464-8601, Japan \\ e-mail: yuri.f@nagoya-u.jp \\ 2 Department of Physics, Nagoya University, Furo-cho, Chikusa-ku, Nagoya, Aichi 464-8602, Japan \\ 3 Division of Science, National Astronomical Observatory of Japan, 2-21-1, Osawa, Mitaka 181-8588, Tokyo, Japan
}

Received 26 November 2019 / Accepted 29 January 2020

\begin{abstract}
Context. Several mechanisms have been proposed to explain the formation process of satellite systems, and relatively large moons are thought to be born in circumplanetary disks. Making a single-moon system is known to be more difficult than multiple-moon or moonless systems.

Aims. We aim to find a way to form a system with a single large moon, such as Titan around Saturn. We examine the orbital migration of moons, which change their direction and speed depending on the properties of circumplanetary disks.

Methods. We modeled dissipating circumplanetary disks with taking the effect of temperature structures into account and calculated the orbital evolution of Titan-mass satellites in the final evolution stage of various circumplanetary disks. We also performed $N$-body simulations of systems that initially had multiple satellites to see whether single-moon systems remained at the end.

Results. The radial slope of the disk-temperature structure characterized by the dust opacity produces a patch of orbits in which the Titan-mass moons cease inward migration and even migrate outward in a certain range of the disk viscosity. The patch assists moons initially located in the outer orbits to remain in the disk, while those in the inner orbits fall onto the planet.

Conclusions. We demonstrate for the first time that systems can form that have only one large moon around giant planet. Our $N$-body simulations suggest satellite formation was not efficient in the outer radii of circumplanetary disks.
\end{abstract}

Key words. planets and satellites: formation - planets and satellites: individual: Titan - planets and satellites: gaseous planets planets and satellites: dynamical evolution and stability - planet-disk interactions

\section{Introduction}

Satellite formation around gas giant planets has been widely discussed in the context of the Galilean moons. The small moons around gas/ice giants in our solar system can also be explained by a tidally spreading solid disk scenario (Charnoz et al. 2010; Crida \& Charnoz 2012; Hyodo et al. 2015) when it is possible to provide the enough material for the initial solid disk. For large moons, such as Io, Europa, Ganymede, and Callisto around Jupiter, and Titan orbiting Saturn, however, it is favorable to have a gaseous circumplanetary disk (CPD) for their formation. Models for CPDs were proposed and the formation of Galilean moons in the disks has been discussed (Canup \& Ward 2002, 2006; Mosqueira \& Estrada 2003a,b; Estrada \& Mosqueira 2006; Sasaki et al. 2010; Ogihara \& Ida 2012; Miguel \& Ida 2016; Fujii et al. 2017; Cilibrasi et al. 2018; Shibaike et al. 2019; Arakawa \& Shibaike 2019). Saving satellites from inward migration and configuring a system in a Laplace resonance are current topics of strong interest. We can find from these studies that it is difficult to form only one large moon. Satellite systems tend to hold multiple large moons or lose all of these moons at the end of the simulations. Although Sasaki et al. (2010) demonstrated that single-moon systems form as a result of population synthesis calculations, it is likely that their method for generating new moon seeds is not appropriate for moon formation (see Sect. 5 of Ogihara \& Ida (2012)).

We aim to find a way to form a system with only one large moon in a CPD. The feasibility of satellitesimal forma- tion has been examined by Shibaike et al. (2017) and delivery of solid matereal to a CPD has been discussed by Fujita et al. (2012), Tanigawa et al. (2014), Suetsugu \& Ohtsuki (2017), and Ronnet et al. (2018). We focus on the later stage of the satellite formation and investigate the orbital evolution of the moons in a dissipating CPD to determine the final appearance of the system.

\section{Disk model}

We derived 1D models of CPDs considering the effect of opacities that can change not only the temperature but also the surface density structures of the disks. The surface density, $\Sigma$, is determined by the balance of the mass infall from the parental protoplanetary disk (PPD) and the viscous evolution of the CPD as described in the Fig. 1, and its time evolution can be calculated with the following diffusion equation:

$\frac{\partial \Sigma}{\partial t}=\frac{1}{r} \frac{\partial}{\partial r}\left[3 r^{1 / 2} \frac{\partial}{\partial r}\left(r^{1 / 2} v \Sigma\right)\right]+f$,

where $r$ is the radial distance from the planet, $v$ is the kinematic viscosity coefficient, and $f$ is the mass flux from the PPD that has the Keplerian angular momentum of the corresponding radii of the CPD. Tanigawa et al. (2012) show $f \propto r^{-1}$ and we adopt $f=5.5 \times 10^{-5}\left(r / R_{\mathrm{p}}\right)^{-1} \mathrm{~g} \mathrm{~cm}^{-2} \mathrm{~s}^{-1}$, where the radius of the planet $R_{\mathrm{p}}$ is the Saturnian radius, until the disk starts to dissipate. We assume the planet is located at $9.5 \mathrm{AU}$ of the minimum mass solar nebula (MMSN; Hayashi 1981) and give $f$ only within 


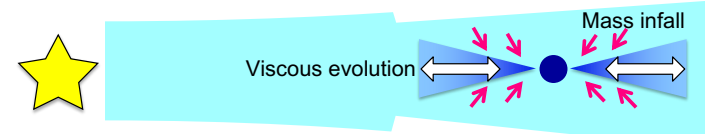

Fig. 1. Schematic picture of how to determine the surface density; viscous evolution of the CPD balances with the mass infall from the PPD.

$r_{\text {infall }}=45 R_{\mathrm{p}}(0.04$ Hill radii) in this work (see Fujii et al. 2017, for the details). The final configuration of the system is not sensitive to the choice of $r_{\text {infall }}$, and changing it to $30 R_{\mathrm{p}}$ or $60 R_{\mathrm{p}}$ does not change the result. We set the size of the CPD as $\sim 0.7$ Hill radii.

The disk midplane temperature, $T_{\mathrm{c}}$, is calculated by

$\frac{\partial T_{\mathrm{c}}}{\partial t}=\frac{2\left(Q_{+}-Q_{-}\right)}{c_{\mathrm{p}} \Sigma}-v_{\mathrm{r}} \frac{\partial T_{\mathrm{c}}}{\partial r}$,

where $Q_{+}=(9 / 8) v \Sigma \Omega_{\mathrm{K}}^{2}$ is the viscous heating, $Q_{-}=$ $\sigma(3 / 8 \kappa \Sigma) T_{\mathrm{c}}^{4}$ denotes the radiative cooling, $\sigma$ is the StefanBoltzmann constant, $v_{\mathrm{r}}$ is the radial velocity, and $\kappa$ is the opacity (Cannizzo 1993; Armitage et al. 2001). The opacities employed are provided by Bell \& Lin (1994). We also modeled CPDs by assuming an order of magnitude smaller dust/ice opacities (see Sect. 5). We set the minimum temperature of the disk as $T_{\min }=90 \mathrm{~K}$.

The origin of the angular-momentum transport in a CPD is uncertain. The magnetorotational instability (MRI), which is believed to be one of the sources of the turbulent viscosity in a PPD, is not very effective in a CPD except for the vicinity of the planet (Fujii et al. 2011, 2014; Turner et al. 2014; Keith \& Wardle 2014). Thus, we assume the mass accretion is gentle in a CPD. We leave the origin of the viscosity unspecified and adopt $v=\alpha c_{\mathrm{s}} h$ with viscous parameter $\alpha$ of Shakura \& Sunyaev (1973), sound speed $c_{\mathrm{s}}$ and pressure scale height of the disk $h$.

With a fixed viscous parameter, the CPD settles into a steady state as long as it has the constant infall. For example, the purple lines in Fig. 2 show the steady-state disk structure for $\alpha=10^{-4}$. Unlike Fujii et al. (2017), we do not introduce a scaling factor to modify the local surface density of the PPD because this parameter is coupled with the viscous parameter when we derive the steady state. The value of $f$ corresponds to the surface density of the PPD that is reduced to $10 \%$ of the MMSN. In order to investigate the final stage of the satellite formation, we calculate the time evolution of CPDs after the mass inflow from the PPD has terminated ( $f$ is set to be zero). The manner of the disk dissipation in the case with $\alpha=10^{-4}$ is described in Fig. 2. We assume the timescale of halting the infall is small enough.

\section{Orbital evolution of moons}

We calculated the orbital evolution of satellites in the dissipating CPDs from Sect. 2 to determine the configuration of the system. The orbital radius of a moon is determined by

$\frac{\mathrm{d} r}{\mathrm{~d} t}=\beta(1+0.04 \mathrm{~K})^{-1} \frac{M_{\mathrm{m}}}{M_{\mathrm{p}}} \frac{\Sigma r^{2}}{M_{\mathrm{p}}}\left(\frac{r \Omega_{\mathrm{K}}}{c_{\mathrm{S}}}\right)^{2} r \Omega_{\mathrm{K}}$,

where $M_{\mathrm{m}}$ and $M_{\mathrm{p}}$ are the mass of the moon and planet, respectively, $\beta$ is a migration parameter that determines the
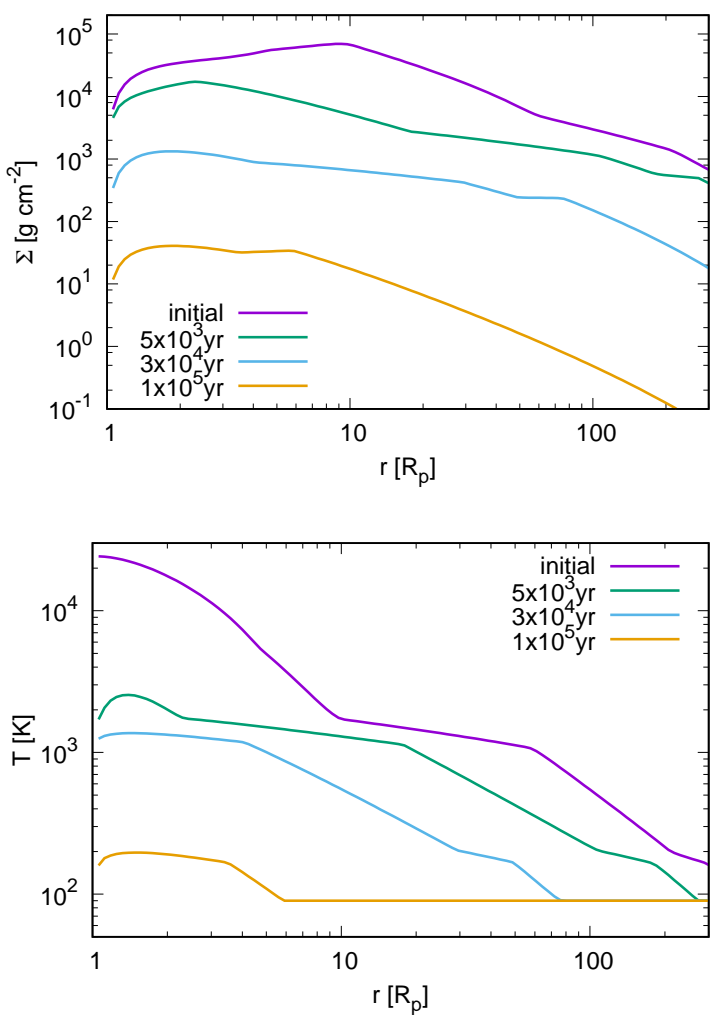

Fig. 2. Time evolution of the surface density (top) and temperature (bottom) structure of the dissipating CPD with $\alpha=10^{-4}$. The time is measured from when the mass infall has terminated.

direction and speed of the migration (Paardekooper et al. 2011; Kretke \& Lin 2012; Ogihara et al. 2015). The factor $(1+0.04 \mathrm{~K})$ connects type I and type II migration using $K=\left(M_{\mathrm{m}} / M_{\mathrm{p}}\right)^{2}(\mathrm{~h} / \mathrm{r})^{-5} \alpha^{-1}$ (Kanagawa et al. 2015, 2018; Ogihara \& Hori 2019). The migration parameter depends on the radial dependence of the surface density and the temperature of the disk. When $\beta$ is positive, the moon migrates outward. Otherwise, the direction of the satellite migration is toward the planet. We used the formula of Paardekooper et al. (2011) for $\beta^{1}$, which is also summarized in Ogihara et al. (2015). The migration parameter also depends on the eccentricity $e$ and inclination $i$ of an orbit (Bitsch \& Kley 2010), and we assumed $e=i=0$ in this section. Those dependences (Cresswell \& Nelson 2008) are, however, taken into account in the Sect. 4. The map of $\beta$ for satellites of 0.01-10 Titan mass in the disk with $\alpha=10^{-4}$ is shown in Fig. 3. The red patches in the figure indicate the parameter space with outward migration. The patch located at $r=\sim 20-100 R_{\mathrm{p}}$ is produced by the shallow and steep radial dependence of $\Sigma$ and $T$ characterized by the dust opacity. The outer patch located at $r>100 R_{\mathrm{p}}$ reflects the opacity due to ice. We can see that Titan-mass satellites captured in these patches escape from inward migration.

Figure 4 demonstrates the orbital evolution of moons with Titan mass. We note that the plots are meant for showcasing the individual evolution of each of the moons in the disks, and therefore, the gravitational interactions with other moons are not considered in the calculation. With $\alpha=10^{-3}$, as shown in Fig. 4a, satellites tend to survive until the disk disappears; thus, if there are multiple moons at the beginning, the system would keep the moons for a longer time period. This is partially because of the

1 See the Appendix A of Bitsch \& Kley (2011) about the factor 4 of the thermal diffusivity. 


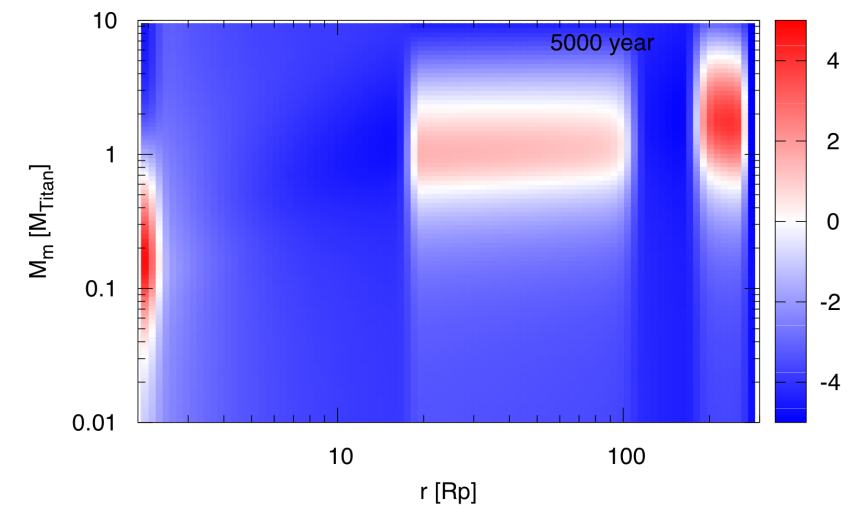

Fig. 3. Migration map for a disk with $\alpha=10^{-4}$ at 5000 years after the dissipation has started (corresponding to the green lines in Fig. 2). The color bar shows the value of $\beta /(1+0.04 \mathrm{~K})$. The direction of a migration is inward/outward in the blue/red region.

saturation of the corotation torque and also because of the quick dissipation of the disk. On the other hand, all the moons are lost as a consequence of the inward migration in the case of $\alpha=10^{-5}$ (Fig. 4c). In this case, the timescales of the migration in the inner orbits are much shorter than in the case of $\alpha=10^{-3}$ and it takes longer until the disk disappears. The most interesting parameter is $\alpha=10^{-4}$, with which the moons in the inner orbits fall onto the central planet but others in the outer orbits remain in the disk as shown in Fig. $4 \mathrm{~b}$. The patch of $\beta>0$ created by the dust opacity prevent moons from moving toward the planet, while inner orbits are cleared. Whether the surviving satellites remain in a couple of tens Saturnian radii is determined by the timing of the disk dissipation.

How many satellites can form and exist at one time depends not only the CPD structure but also the manner and amount of the solid supply. We found that even if many moons form in the disk, most of them would be lost and only a couple of them remain when the CPD has been dissipated. Through this mechanism, we can possibly form a single-moon system unless the satellite formation in the large orbit is very efficient. In order to further investigate the possibility, we carry out $N$-body simulations in the next section. Although it might be challenging to create many satellites in a CPD, we insert relatively large number of moons as our goal is to make a single-moon system.

\section{4. $\mathbf{N}$-body simulations}

In the calculations shown in the previous section, the mutual gravitational interaction between moons is not considered. In this section, we perform simulations that include the $N$-body interaction and examine whether single-moon systems can form with the initial setting supposing optimistically many moons can form before the CPD starts to dissipate. We do not include moons that form during the run because we assume there is no supply of solid when gas inflow from the PPD has finished.

Figure 5 shows the time evolution of the semimajor axis. In Fig. 5a, seven moons with one Titan mass are initially placed between $5 R_{\mathrm{p}}$ and $40 R_{\mathrm{p}}$ with separations of 6 mutual Hill radii. As seen in Fig. 4, while inner moons migrate inward and fall onto the planet, a moon in an outer orbit undergoes outward migration and avoids falling onto the planet. Finally, the moon remains in the outer orbit and a single-moon system forms. Figure $5 \mathrm{~b}$ shows simulation result that starts with nine moons with 0.5 Titan masses. We also see that inner moons fall onto the planet and a single moon with a Titan mass remains.
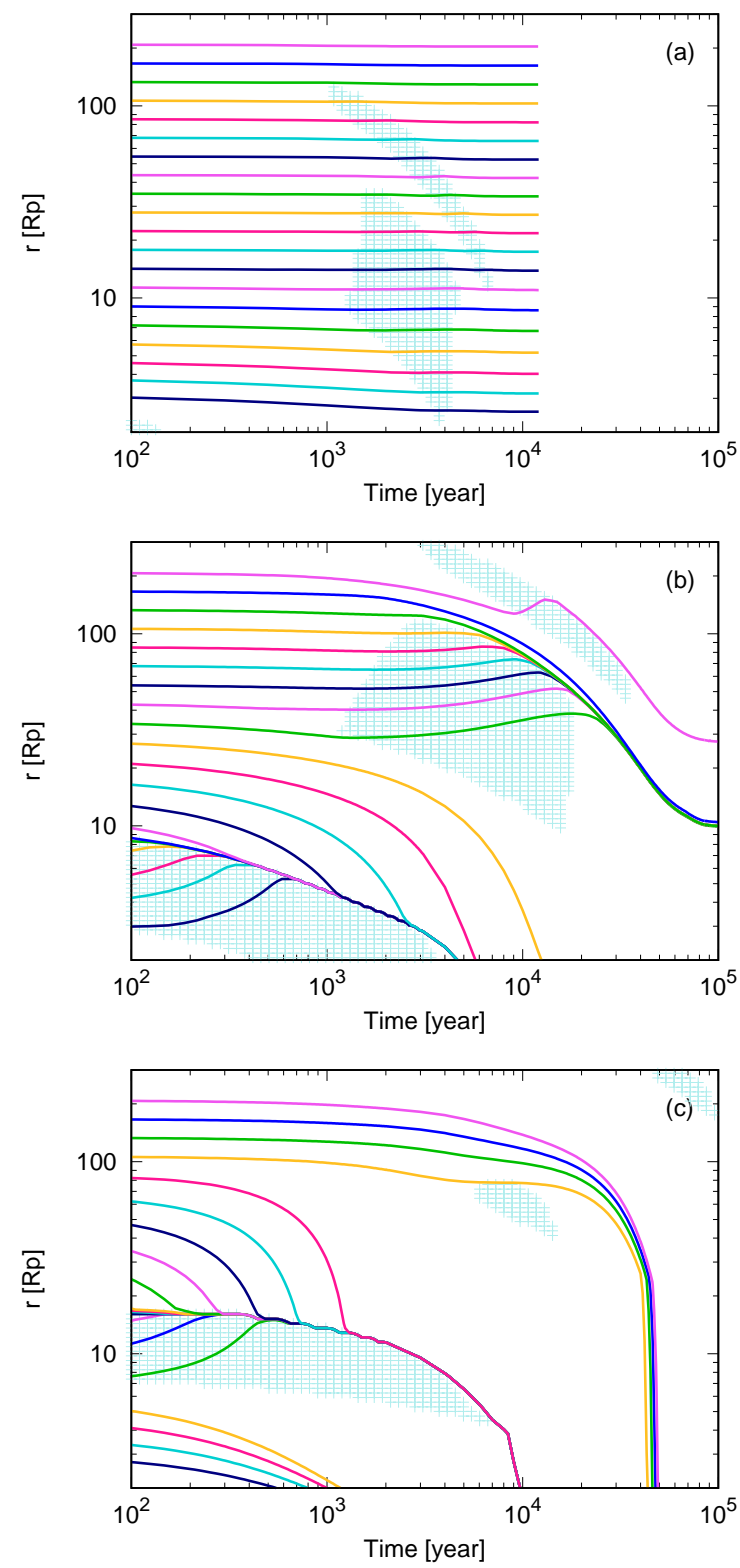

Fig. 4. Orbital evolution of individual moons since the disk dissipation started with various initial locations in the case where $\alpha=10^{-3}, \alpha=$ $10^{-4}$ and $\alpha=10^{-5}$ from top to bottom. The vertical axis is normalized by the planet radius. The shaded areas show when and where $\beta>0$ for Titan mass moons, which correspond to the red regions in Fig. 3. The mutual interactions of satellites are not considered in these plots.

Although multiple moons remain in previous $N$-body simulations (e.g., Canup \& Ward 2006; Ogihara \& Ida 2012), we first demonstrate that single-moon systems can form under some conditions. As already stated above, the number of remaining moons depends on the property of CPD and the solid distribution. For example, when we start simulations with moons that extend beyond $40 R_{\mathrm{p}}$, the number of final moons tends to increase. This is consistent with the hypothesis that regular moons grow in the inner region, which justifies our assumption of initial distribution of moons.

\section{Discussions and conclusions}

We demonstrated the qualitative pathway to establish a singlemoon system: 
(a)

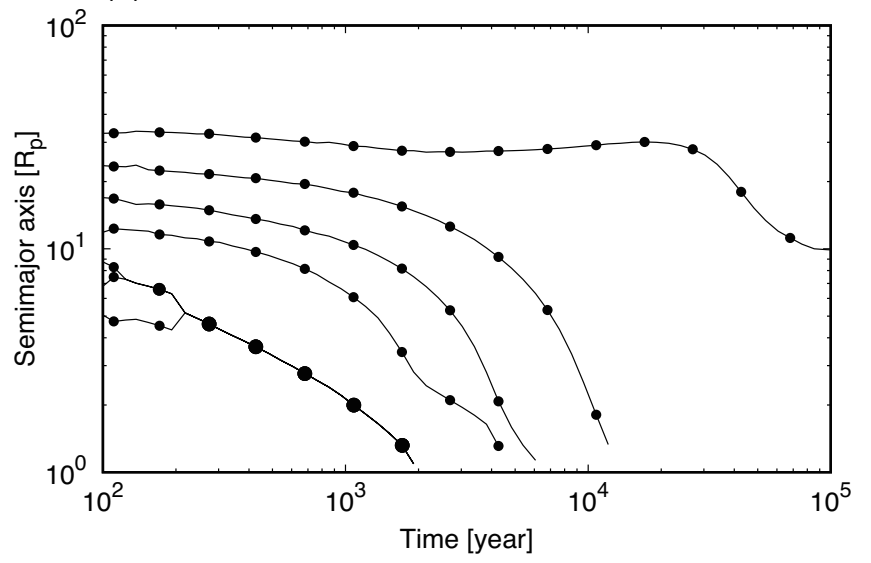

(b)

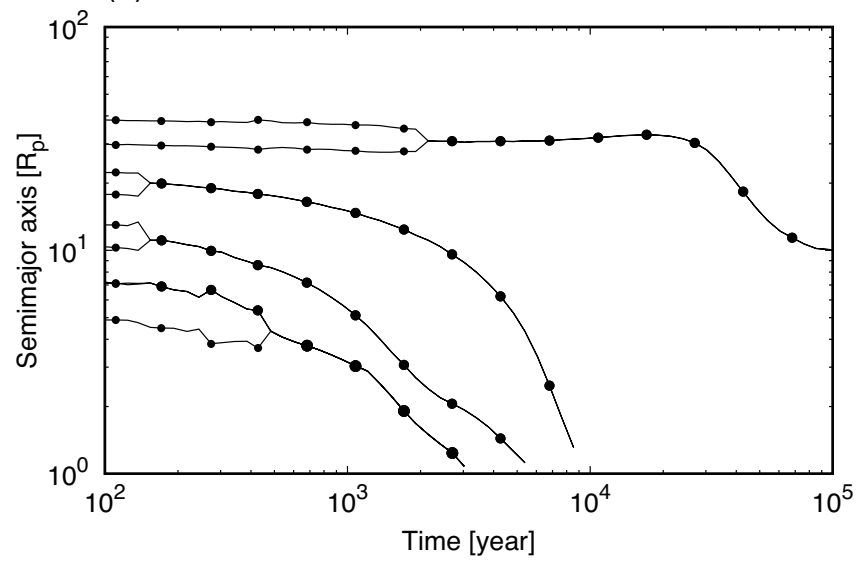

Fig. 5. Results of the N-body simulations. (a) The case with initially seven moons of the Titan mass. (b) The case with nine moons with 0.5 Titan masses. We terminated the simulations when the disk gas has mostly dissipated and no more satellite migration was expected.

1. Moons in the outer orbits are captured in the patch with outward migration.

2. Inner orbits are cleared by type I migration.

3. The disk disappears before the final moon falls onto the planet.

We find that there is a favorable value of the viscous parameter to form a system that has a single Titan-mass satellite; this value is $\alpha=10^{-4}$ in our settings. With $\alpha=10^{-3}$, more than one of the moons tend to survive if they have formed. In the case of $\alpha=10^{-5}$, all the moons would be lost unless the disk dissipation has accelerated. As shown in Fig. 3, the direction of migration changes with the mass of moons, thus the conditions to be a single-moon system differs in the cases for other masses. Other than the migration parameter, the timescale for the disk dissipation is also an important factor.

We employed the $\alpha$-disk model for simplicity, and assumed the value of $\alpha$ as a constant both in time and space. Even in a dissipating CPD, the MRI may not contribute (see Fig. 6 of Fujii et al. 2014), however, other mechanisms may vary the dynamical evolution of the disk. In such a case, the condition to obtain a single-moon system and the location of the moon would be modified.

We also calculated cases with an order of magnitude smaller dust and ice opacities compared with those of Bell \& Lin (1994). Of course, the disk surface-density and temperature structures are modified but the tendency of the orbital evolution of the moons in the disks were similar to the case with the original opacity. In the lower opacity case, the disk should dissipate a little too quickly.

Acknowledgements. We thank the anonymous referee for useful comments. YIF was supported by the JSPS KAKENHI Grant Number JP18K13604 and Start-up grant of Building of Consortia for the Development of Human Resources in Science and Technology from Ministry of Education, Culture, Sports, Science, and Technology (MEXT). Numerical computations were in part carried out on PC cluster at Center for Computational Astrophysics, National Astronomical Observatory of Japan.

\section{References}

Arakawa, S., \& Shibaike, Y. 2019, A\&A, 629, A106 Armitage, P. J., Livio, M., \& Pringle, J. E. 2001, MNRAS, 324, 705 Bell, K. R., \& Lin, D. N. C. 1994, ApJ, 427, 987 Bitsch, B., \& Kley, W. 2010, A\&A, 523, A30

Bitsch, B., \& Kley, W. 2011, A\&A, 536, A77

Cannizzo, J. K. 1993, ApJ, 419, 318

Canup, R. M., \& Ward, W. R. 2002, AJ, 124, 3404

Canup, R. M., \& Ward, W. R. 2006, Nature, 441, 834

Charnoz, S., Salmon, J., \& Crida, A. 2010, Nature, 465, 752

Cilibrasi, M., Szulágyi, J., Mayer, L., et al. 2018, MNRAS, 480, 4355

Cresswell, P., \& Nelson, R. P. 2008, A\&A, 482, 677

Crida, A., \& Charnoz, S. 2012, Science, 338, 1196

Estrada, P. R., \& Mosqueira, I. 2006, Icarus, 181, 486

Fujii, Y. I., Okuzumi, S., \& Inutsuka, S. 2011, ApJ, 743, 53

Fujii, Y. I., Okuzumi, S., Tanigawa, T., \& Inutsuka, S. 2014, ApJ, 785, 101

Fujii, Y. I., Kobayashi, H., Takahashi, S. Z., \& Gressel, O. 2017, AJ, 153, 194

Fujita, T., Ohtsuki, K., \& Tanigawa, T. 2012, Lunar and Planetary Institute Science Conference Abstracts, 43, 1378

Hayashi, C. 1981, Prog. Theor. Phys. Suppl., 70, 35

Hyodo, R., Ohtsuki, K., \& Takeda, T. 2015, ApJ, 799, 40

Kanagawa, K. D., Tanaka, H., Muto, T., Tanigawa, T., \& Takeuchi, T. 2015, MNRAS, 448, 994

Kanagawa, K. D., Tanaka, H., \& Szuszkiewicz, E. 2018, ApJ, 861, 140

Keith, S. L., \& Wardle, M. 2014, MNRAS, 440, 89

Kretke, K. A., \& Lin, D. N. C. 2012, ApJ, 755, 74

Miguel, Y., \& Ida, S. 2016, Icarus, 266, 1

Mosqueira, I., \& Estrada, P. R. 2003a, Icarus, 163, 198

Mosqueira, I., \& Estrada, P. R. 2003b, Icarus, 163, 232

Ogihara, M., \& Hori, Y. 2019, ApJ, submitted

Ogihara, M., \& Ida, S. 2012, ApJ, 753, 60

Ogihara, M., Kobayashi, H., Inutsuka, S.-I., \& Suzuki, T. K. 2015, A\&A, 579, A65

Paardekooper, S.-J., Baruteau, C., \& Kley, W. 2011, MNRAS, 410, 293

Ronnet, T., Mousis, O., Vernazza, P., Lunine, J. I., \& Crida, A. 2018, AJ, 155, 224

Sasaki, T., Stewart, G. R., \& Ida, S. 2010, ApJ, 714, 1052

Shakura, N. I., \& Sunyaev, R. A. 1973, A\&A, 24, 337

Shibaike, Y., Okuzumi, S., Sasaki, T., \& Ida, S. 2017, ApJ, 846, 81

Shibaike, Y., Ormel, C. W., Ida, S., Okuzumi, S., \& Sasaki, T. 2019, ApJ, 885, 79

Suetsugu, R., \& Ohtsuki, K. 2017, ApJ, 839, 66

Tanigawa, T., Maruta, A., \& Machida, M. N. 2014, ApJ, 784, 109

Tanigawa, T., Ohtsuki, K., \& Machida, M. N. 2012, ApJ, 747, 47

Turner, N. J., Lee, M. H., \& Sano, T. 2014, ApJ, 783, 14 